\title{
A Monte Carlo Study of Electron Transport in Silicon nMOSFET Inversion Layers
}

\author{
W.-K. SHIH, S. JALLEPALLI, C.-F. YEAP, M. RASHED, C. M. MAZIAR and A. F. TASCH, JR.
}

Microelectronics Research Center, The University of Texas at Austin, Austin, Texas 78712, USA

Monte Carlo simulations of uniform silicon nMOSFET inversion layers have been performed. Excellent agreement between the simulated and experimental transport characteristics has been observed in the region of strong inversion at both $300 \mathrm{~K}$ and $77 \mathrm{~K}$. The contribution to the effective mobility due to individual subbands has been analyzed and qualitatively explained.

\section{INTRODUCTION}

Despite decades of research effort [1], problems associated with carrier transport in the inversion layers of silicon MOSFETs continue to capture the attention of the research community [2-4]. Today's advanced devices introduce further complications with the aggressive scaling of channel length that introduces pronounced non-local effects such as velocity overshoot [4]. In order to investigate and anticipate future device performance issues, simulation tools that rely on physically rather than empirically based models are required. In particular, the demand for including inversion-layer quantization effects in routine device simulation $[5,6]$ has been well appreciated.

We present here Monte Carlo (MC) simulations of uniform silicon nMOSFET inversion layers within and beyond the ohmic regime for various substrate doping levels and gate biases at different temperatures. Within the range of the lateral electric-field commonly seen in the linear region of silicon nMOSFETs, transport of the two-dimensional electron gas (2DEG) is well described by a two-dimensional multi-subband Boltzmann transport equation (BTE.)
Particle simulations such as the MC technique $[3,7]$ provide a direct solution to the BTE and are especially indispensable in the study of high-field and non-local effects.

\section{THEORY}

The subband structure used in this work is a modified version of that developed in [3], where the effectivemass approximation (EMA) is based on the bulk nonparabolic relation, $\mathrm{E}(\mathbf{K})=\gamma_{\text {bulk }}(\mathbf{K})\left(1+\alpha \gamma_{\text {bulk }}(\mathbf{K})\right), \gamma_{\text {bulk }}$ being the parabolic dispersion relation for bulk silicon, $\mathbf{K}$ the 3D wave vector and $\alpha$ the non-parabolicity, which is negative for the lowest valley in the conduction band of silicon. Starting with a different bulk nonparabolic $\mathrm{E}(\mathbf{K})$ relation $\mathrm{E}(\mathbf{K})(1+\alpha \mathrm{E}(\mathbf{K}))=\gamma_{\text {bulk }}(\mathbf{K}), \alpha$ being $0.5 \mathrm{eV}^{-1}$, the dispersion relation $\mathrm{E}_{v}(\mathbf{k})$ for the $v$ th subband satisfies:

$$
\begin{aligned}
& \left(\mathrm{E}_{v}(\mathbf{k})-\left\langle\mathrm{E}_{\mathrm{c}}\right\rangle_{v}\right)\left[1+\alpha\left(\mathrm{E}_{v}(\mathbf{k})-\left\langle\mathrm{E}_{\mathrm{c}}\right\rangle_{v}\right)\right] \\
& =\gamma_{2 \mathrm{D}}(\mathbf{k})+\varepsilon_{v}-\left\langle\mathrm{E}_{\mathrm{c}}\right\rangle_{v}
\end{aligned}
$$

where 


$$
\gamma_{2 \mathrm{D}}=\frac{\hbar^{2} \mathbf{k}_{\mathrm{x}}^{2}}{2 \mathrm{~m}_{\mathrm{x}}^{*}}+\frac{\hbar^{2} \mathbf{k}_{\mathrm{y}}^{2}}{2 \mathrm{~m}_{\mathrm{y}}^{*}}
$$

$\epsilon_{v}$ and $\zeta_{v}$ are the eigenenergy and eigenfunction of the zeroth-order Schroedinger equation:

$$
\left[-\frac{\hbar^{2}}{2 \mathrm{~m}_{\mathrm{z}}^{*}} \frac{\mathrm{d}^{2}}{\mathrm{~d} z^{2}}+\mathbf{E}_{\mathrm{c}}(\mathbf{z})\right] \zeta_{\mathrm{v}}(\mathbf{z})=\varepsilon_{\mathrm{v}} \varsigma_{\mathrm{v}}(\mathbf{z}),
$$

and \langle\rangle$_{v}$ denotes the expectation value taken over $\zeta_{v}$. In the above equations, $\mathrm{E}_{\mathrm{c}}$ is the energy at the conduction band edge that depends only on $\mathrm{z}$, the coordinate perpendicular to the $\mathrm{Si} / \mathrm{SiO}_{2}$ interface, and $\mathbf{k}$ is the $2 \mathrm{D}$ wave vector in the $x-y$ plane. The density of states at energy $E$ of the $v$ th subband and the nth valley, including the spin degeneracy, is

$$
\begin{aligned}
D_{\mathrm{n}, v}(\mathrm{E})=\frac{\mathrm{m}_{\mathrm{d}}}{\pi \hbar^{2}}\left[1+2 \alpha\left(\mathrm{E}-\left\langle\mathrm{E}_{\mathrm{c}}\right\rangle_{\mathrm{n}, v}\right)\right] \\
\quad \theta\left(\mathrm{E}-\mathrm{E}_{\mathrm{n}, \mathrm{v}}(\mathrm{k}=0)\right)
\end{aligned}
$$

where $m_{d}$ is the $2 \mathrm{D}$ density of states effective mass.

Phonon and surface roughness scattering have been included in the present work. For phonon scattering, the formalism for electron scattering with bulk acoustic and optical phonons [8] is used. The coupling constant between electron and intravalley acoustic phonons is assumed to be isotropic and an effective coupling constant is used to reduce the computational burden. For intervalley phonon scattering, constant phonon dispersion relations have been employed for optical modes and intervalley acoustic phonon energies have been taken as constants. Intravalley optical phonon processes are ignored. Since in the past [9] the effect due to the surface vibration modes, the socalled "surfons", has been found to be small, scattering between inversion layer electron and surface phonons is also ignored. Finally, the simple model due to Cheng $[7,10]$ has been applied in treating the surface roughness scattering.

\section{SIMULATIONS}

The physical models described in the previous section have been implemented in UT-MINIMOS [11, 12], a version of MINIMOS developed at the University of
Texas that encompasses drift-diffusion (DD), hydrodynamic (HD) and MC modeling tools. Since we are interested in uniform inversion layers, long-channel devices with zero source-drain bias and uniform substrate doping concentrations are used. For a given substrate doping level, the gate bias is ramped in the region of strong inversion. At each gate bias step, a slice along the depth direction is selected in the middle of the channel, where the electron subband structure in the inversion layer is calculated by selfconsistently solving the one-dimensional Schroedinger and Poisson equations. Single-particle MC simulation is subsequently performed. A sufficient number of subbands and an upper energy boundary are chosen to avoid running out of density of states during the entire simulation. The Pauli exclusion principle has been included.

\section{RESULTS AND DISCUSSION}

The universal relationship between the effective mobility and the transverse effective field has been reproduced (see Fig. 1). Good agreement between the simulated and experimental low-field mobility has

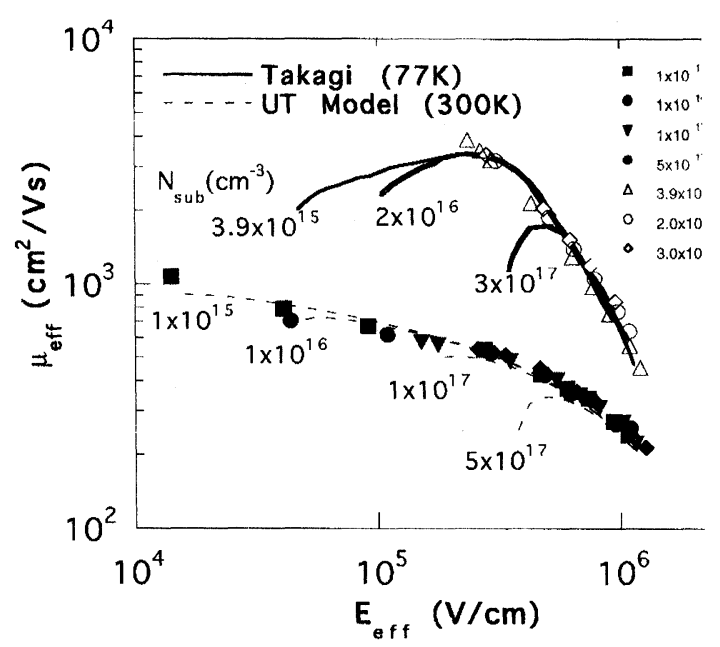

FIGURE 1 Calculated low-field effective mobility (solid and empty symbols for $300 \mathrm{~K}$ and $77 \mathrm{~K}$, respectively) versus the transverse effective field for substrate doping ranging from $1 \times 10^{15}$ to $5 \times 10^{17} \mathrm{~cm}^{-3}$ compared to experimental data $(77 \mathrm{~K})[13]$ and the UT mobility model $(300 \mathrm{~K})$ [2] 
been achieved for both $300 \mathrm{~K}$ and $77 \mathrm{~K}$. The simulated results are compared with the experimental work of Takagi [13] at 77K and with the UT mobility model [2] at 300K. The UT mobility model has been well characterized with a large number of devices of varying structures and industrial sources. As such it is used as the best representation of a large body of experimental work.

The fractional population and the average mobility of electrons in the lowest primed and unprimed subbands are illustrated in Fig. 2. At low $\mathrm{E}_{\text {eff }}$, the sub-

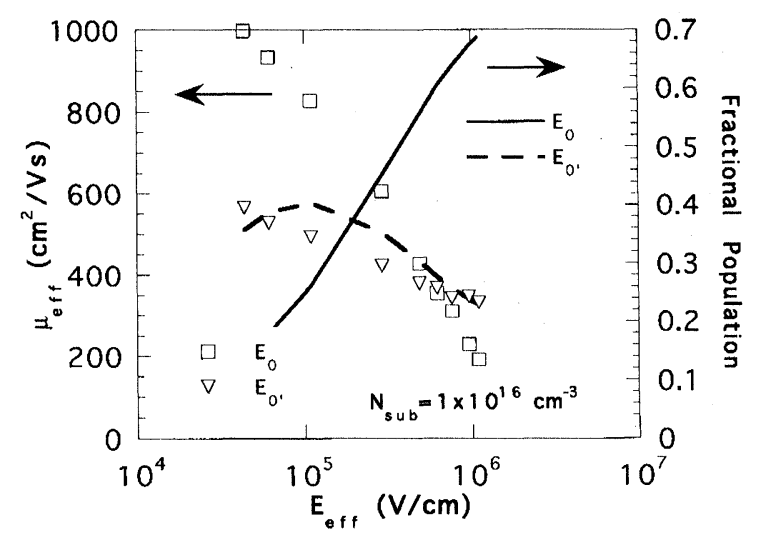

FIGURE 2 Mobility of electrons in the lowest unprimed and primed subbands (symbols) and their population fraction (lines) at $300 \mathrm{~K}$. The higher population and lower mobility of the primed subband at low $\mathrm{E}_{\mathrm{eff}}$ is due to its larger density of states and higher conductivity effective mass. At high $\mathrm{E}_{\mathrm{eff}}$, the subband spacing causes the population of the unprimed subband to dominate

band spacing is comparable to the thermal energy and the density of states plays a dominant role in determining the subband populations. As $\mathrm{E}_{\mathrm{eff}}$ increases, the subband spacing increases and the fractional population in the ground subband increases accordingly. This results in the crossing between the fractional populations plotted against $\mathrm{E}_{\mathrm{eff}}$. Due to the stronger surface roughness scattering in the unprimed subband, the effective mobility of the unprimed subband demonstrates a higher sensitivity to $\mathrm{E}_{\text {eff }}$ compared to that of the primed subband. Fig. 3 shows the electron drift velocity as a function of the lateral electric-field for various $\mathrm{E}_{\mathrm{eff}}$. The calculated inversion layer saturation velocity is found to be independent of the trans-

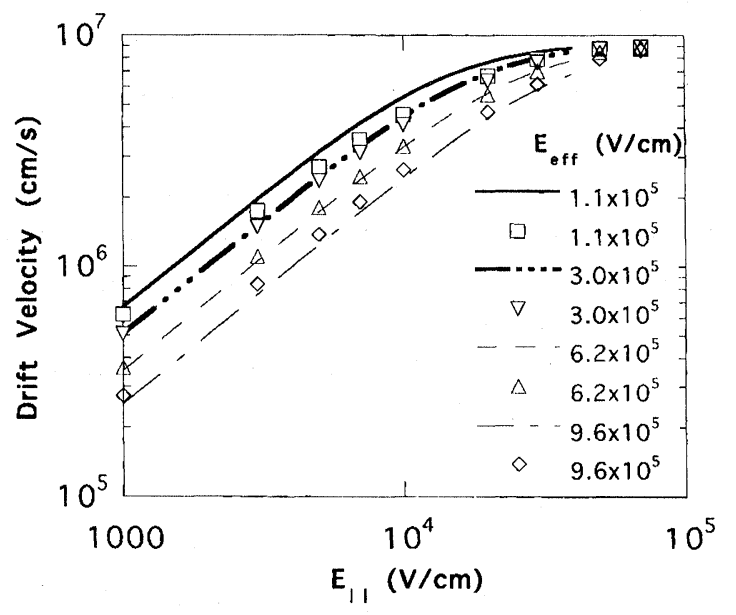

FIGURE 3 Calculated drift velocity (symbols) compared to values predicted by the UT-mobility model (lines) [2] at 300K. The saturation velocity is observed to be independent of $E_{\text {eff }}$

verse effective-field and agrees well with experimental data as described by the UT mobility model.

Microscopic information such as particle energy-distribution has been extracted during the MC simulation. The distribution at low lateral field resembles the density-of-state weighted Maxwell-Boltzmann distribution and spreads into a higher energy domain at moderately-high lateral-field, indicating the necessity of including either more subbands or a classical simulation domain if the tail of the energy distribution is desired.

As the inversion-layer electrons are redistributed among the subbands at high lateral-field, the spatial charge concentration is expected to change since the envelope functions of higher subbands spread more into the bulk silicon than those of the lower subbands. We have used a post-MC Schroedinger-Poisson loop to investigate this high lateral-field induced effect. Instead of the equilibrium Fermi distribution, the fractional population in each subband collected during the MC stage is used in the post-processing loop to selfconsistently solve the Schroedinger and Poisson's equations. The new $\mathrm{E}_{\mathrm{eff}}$ thus calculated has been found to be different from the original $\mathrm{E}_{\mathrm{eff}}$ by less than $3 \%$. This justifies the way data are presented and compared in this work. 


\section{SUMMARY}

A Monte Carlo tool that accurately reproduces and explains the experimental universal mobility curve at different temperatures has been developed. Its capability of capturing high-field transport characteristics in uniform silicon nMOSFET inversion layer has been confirmed. The Monte Carlo tool also provides access to important but experimentally unavailable microscopic information.

\section{Acknowledgements}

This work was sponsored, in part, under the Joint Services Electronics Program, Contract Number F49620-95-C-0045, by the Semiconductor Research Corporation, by Motorola and by the Texas Advanced Technology Program. The United States Government is authorized to reproduce and distribute reprints for governmental purposes notwithstanding any copyright notation hereon.

\section{References}

[1] T. Ando et al., Rev. Mod. Phys., vol. 54, p. 437, 1982.

[2] H. Shin et al., Solid State Electron., vol. 34 , no. 6, p. 545, 1991.

[3] M. V. Fischetti et al., Phys. Rev. B, vol. 48, no. 4, p. 2244, 1993.

[4] F. Assaderaghi et al., IEDM Tech Dig., p. 479, 1994.

[5] S. Hareland et al., to be published.

[6] M. J. van Dort, et al., Solid State Electron., vol. 37, no. 3, p. 435, 1994.

[7] C. Jungemann et al., Solid State Electron., vol. 36, p.. 1529, 1993.

[8] D. K. Ferry, Surf. Sci., vol. 57, p. 218, 1976. P. J. Price, Anls. Phys. vol. 133, p. 217, 1981.

[9] H. Ezawa et al., Surf. Sci., vol. 58, p.25, 1976.

[10] Y. C. Cheng, Surf. Sci., vol. 27, p. 663, 1971.

[11] C.-F. Yeap et al., NUPADS V, p. 15-18, 1994.

[12] X. L. Wang et. al., J. Appl. Phys., Vol. 73, No. 7, p. 3339, 1993.

[13] S. Takagi, et al., IEEE Trans. on Elec. Devices, vol. 41, no.12, p. 2357, 1994. 

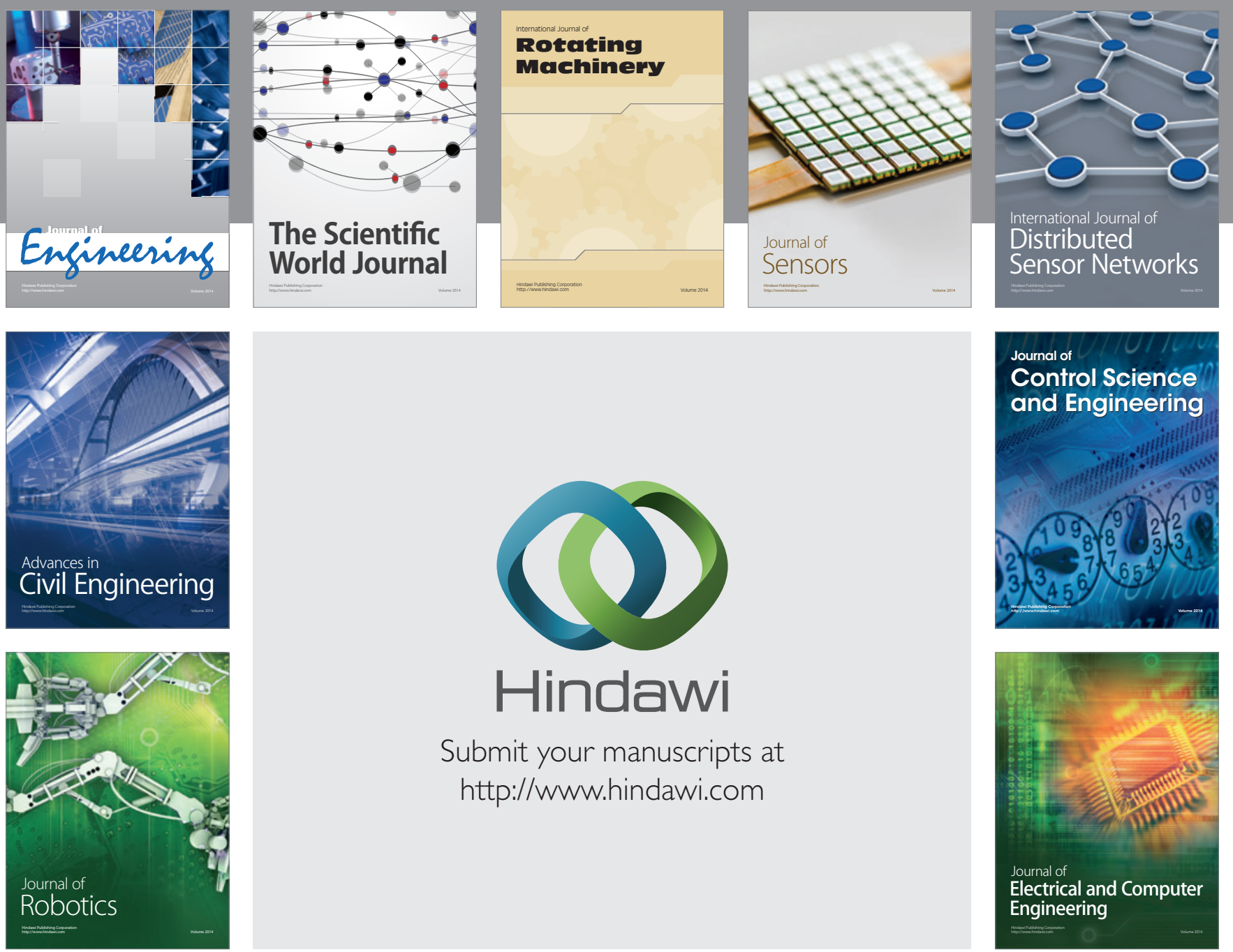

Submit your manuscripts at

http://www.hindawi.com
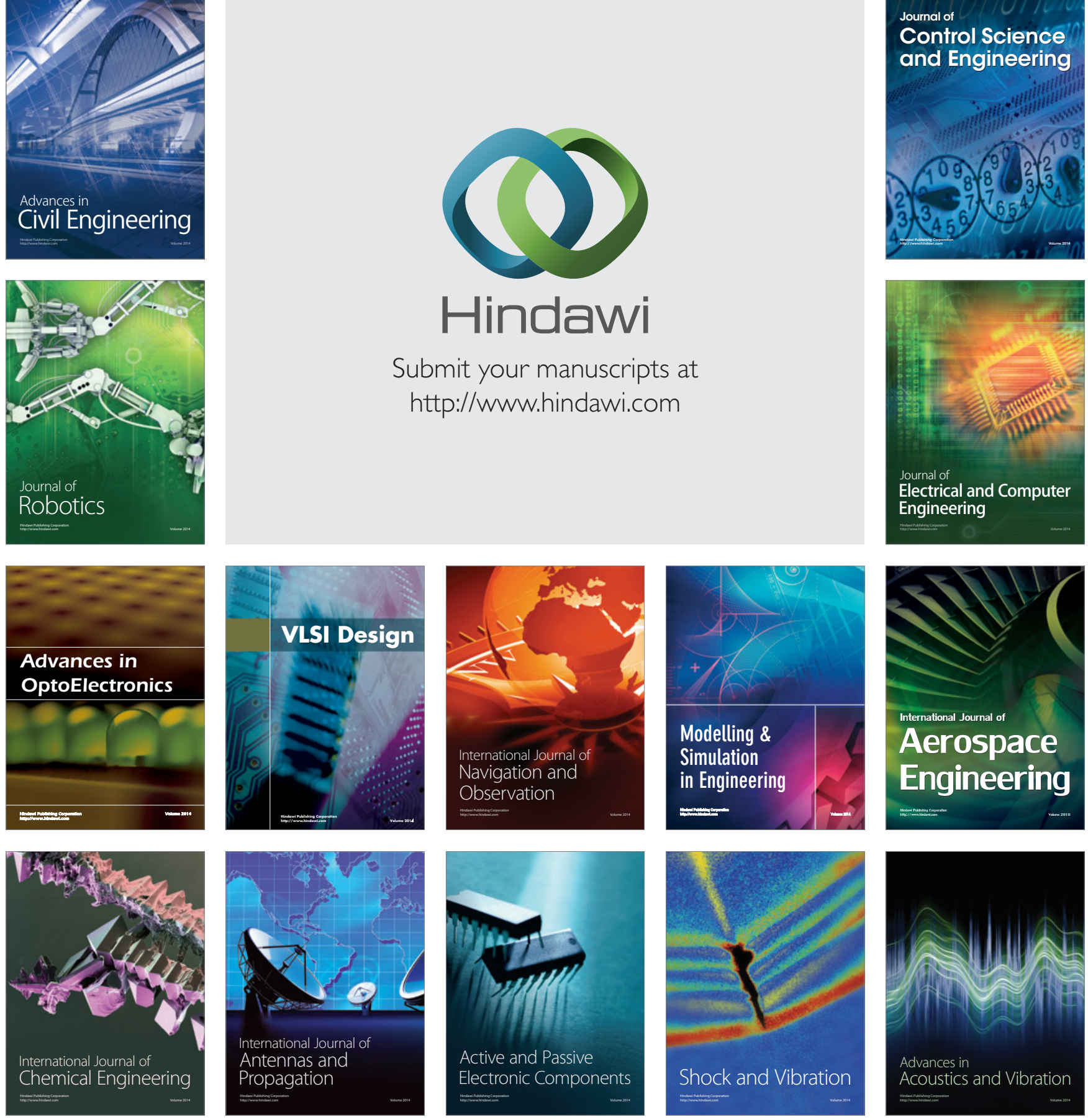